\title{
Genetic diversity of taraxacum germplasm revealed by sequence-related amplified polymorphism (SRAP) analysis
}

\author{
Zhigang $\mathrm{Wu}^{1,2}$, Wei Ning ${ }^{1 *}$, Yaosheng Wang ${ }^{3}$, Shuangshuang $\mathrm{Lv}^{4}$, Yabo Zhao ${ }^{1}$ and Xin Zhao ${ }^{1}$ \\ ${ }^{1}$ College of Horticulture, Shenyang Agricultural University, Shenyang, 110886, China. \\ ${ }^{2}$ Floricultural Research Institute, Liaoning Academy of Agricultural Sciences. \\ ${ }^{3}$ Department of Agriculture and Ecology, Faculty of Life Sciences, University of Copenhagen, Højbakkegård Allé 13, DK- \\ 2630 Taastrup, Denmark. \\ ${ }^{4}$ Institute of Analysis, Shenyang Agricultural University, Shenyang 110886, China.
}

Accepted 22 June, 2011

\begin{abstract}
Sequence-related amplified polymorphism (SRAP) and morphological markers were employed to determine the genetic diversity and relations among 11 population of taraxacum in northeast of China. Data on 34 morphological traits were collected and analyzed. A total of 795 polymorphic SRAP's bands were scored with 20 combinations of primers. The genetic relationships analyzed with un-weighed pairgroup method with arithmetic mean (UPMGA) showed that 11 population of taraxacum were grouped into three clusters. The results reveal by SRAP molecular markers were consistent with those based on the agronomic traits, suggesting that SRAP markers could be used in the taxonomic analysis of taraxacum germplasm.
\end{abstract}

Key words: Taraxacum, sequence-related amplified polymorphism (SRAP), genetic diversity, un-weighed pairgroup method with arithmetic mean (UPMGA).

\section{INTRODUCTION}

Taraxacum is one of the important categories in Compositae and currently, there are more than 2000 species of taraxacum naturalized worldwide (Ge, 1998). China has 70 species and 1 variant. Among them, there are 19 species, 1 variant and 3 forms in northeast of China ( $\mathrm{Li}$, 2004). Taraxacum in China could be used as the traditional Chinese medicine, having sound anti-infec-tion effect, according to the Chinese Pharmacopoeia of 2010. Obviously, the species of taraxacum from different regions may have diverse components account-ing for the effectiveness and stability as medicine. Therefore, it is urgent and crucial that scientific systems of sorting, identifying and evaluating the germplasm resource of

Corresponding author. E-mail: synw_01@163.com. Tel: +8624-88760236. Fax: +86-24-31025677.

Abbreviations: SRAP, Sequence-related amplified polymorphism. taraxacum plants should be established.

Molecular markers are powerful tool to assess the genetic diversity and population structure, because they are plentiful, independent of tissue or environmental effects (Englbrecht et al., 2000; Whitehead et al., 2003; Liu et al., 2006). As one of the DNA-based markers, sequence-related amplified polymorphism (SRAP) is designed to amplify open reading frames (ORFs) ( $\mathrm{Li}$ and Quiros, 2001) based on specially designed primer pairs. The forward primers preferentially amplify exonic regions and the reverse primers preferentially amplify intronic regions and regions with promoters. Compared with other marker systems, SRAP has been demonstrated to be a useful tool for population genetic studies because of its simplicity, reproducibility and disclosure of multiple markers from a single two-primer reaction, when compared with other marker systems ( $\mathrm{Li}$ and Quiros, 2001; Ferriol et al., 2003; Esposito et al., 2007).

Currently, SRAP markers have been successfully used to study genetic diversity and relationships in many 
Table 1. Taraxacum materials and origin of the entire collection.

\begin{tabular}{clll}
\hline Number & Population name & Latin & Origin \\
\hline 1 & Dandong Dandelion & Taraxacum antungense & Shenyang Liaoning \\
2 & Liaodong Dandelion & Taraxacum liaotungense & Chaoyang Liaoning \\
3 & Jipian Dandelion & Taraxacum asiaticum & Chaoyang Liaoning \\
4 & Xing'an Dandelion & Taraxacum falcilobum & Chaoyang Liaoning \\
5 & Chaoxian Dandelion & Taraxacum coreanum & Shenyang Liaoning \\
6 & Guangbao Dandelion & Taraxacum lamprolepis & Chaoyang Liaoning \\
7 & Tujian Dandelion & Taraxacum sinomongolicum & Chifeng Neimeng \\
8 & Yaoyong Dandelion & Taraxacum officinale & Panshi Jilin \\
9 & Menggu Dandelion & Taraxacum mongolicum & Chaoyang Liaoning \\
10 & Juanbao Dandelion & Taraxacum urbanum & Benxi Liaoning \\
11 & Dongbei Dandelion & Taraxacum ohwianum & Dandong Liaoning \\
\hline
\end{tabular}

Table 2. Primers sequence used in this study.

\begin{tabular}{llll}
\hline Code & Forward primers sequence & Code & Reverse primers sequence \\
\hline ME1 & 5'-TGAGTCCAAACCGGATA-3' & EM1 & 5'-GACTGCGTACGAATTAAT-3' \\
ME2 & 5'-TGAGTCCAAACCGGAGC-3' & EM2 & 5'-GACTGCGTACGAATTTGC-3' \\
ME3 & 5'-TGAGTCCAAACCGGA AT-3' & EM3 & 5'-GACTGCGTACGAATTGAC-3' \\
ME4 & 5'-TGAGTCCAAACCGGACC-3' & EM4 & 5'-GACTGCGTACGAATTTGA-3' \\
& & EM5 & 5'-GACTGCGTACGAATTAAC-3' \\
\hline
\end{tabular}

crops, such as cotton (Lin et al., 2003), corn (Jiang et al., 2007) and some vegetable crops including hot pepper (Ren et al., 2004), Brassica napus (Riaz, 2001), pea (Espostio, 2007), potato (He et al., 2007), eggplant (Mutlu, 2008) and Cucurbita pepo (Ferriol et al., 2003). In addition, SRAP markers were also used on some Chinese herbal medicine including Codonopsis tangshen (Chen et al., 2009), Astragalus root (Qian et al., 2009) and Houttuynia cordata (Zhong et al., 2010). Yet, not much is known about whether SARP could be used on taraxacum. Thus, the objective of this study was to investigate genetic diversity and genetic distance for 11 populations of taraxacum from the northeast of China in order to classify the taraxacum populations on the molecular level and provide precision genetic information for the future breeding program.

\section{MATERIALS AND METHODS}

\section{Plant materials}

A total 11 populations of taraxacum were collected from the northeast of China. The locations for the populations are listed in Table 1.

\section{Morphological traits and data analysis}

Several traits were analyzed in order to characterize the plant morphological diversity. The roots of taraxacum collected were cultivated in June, 2009. Flower, plant, leaf and achene were divided into 34 qualitative characters, which with reference to Flora Northeast, Flora of China and Atlas of higher plants in China. Data were collected from September of 2009 for 36 phenotypic traits. Genetic distances were calculated according to Dice genetic similarity (GS). Phenograms were constructed based on unweighted pair-group method analysis (UPGMA) (Sokal and Michener, 1958) using the NTSYS-pc 2.1 software (Rohlf, 2000).

\section{DNA extraction and SRAP analysis}

Young leaves of taraxacum were harvested in April, 2010 and the genomic DNA was extracted by CTAB method (Wu, 1999). Working stock solutions were made by diluting the samples to $25 \mathrm{mg} \mathrm{l}^{-1}$ and stored at $4^{\circ} \mathrm{C}$ for analysis.

SRAP is a PCR-based marker system with two primes, a forward primer of 17 bases and a reverse primer of 18 bases. The primers were composed by Invitrogen Co. Ltd (Table 2). Each 15 ul PCR reaction mixture consisted of $1.5 \mu \mathrm{l}$ of $10 \times \mathrm{PCR}$ buffer $(25 \mathrm{mM}$ of $\left.\mathrm{MgCl}_{2}\right), 1.2 \mu \mathrm{l}$ dNTPs, $1 \mu \mathrm{l}$ forward and reverse primer $(10 \mathrm{mM}), 0.5$ $\mu \mathrm{l}$ Taq DNA polymerase $(2.5 \mathrm{U} / \mu \mathrm{l})$ and $1 \mu \mathrm{l}$ genomic DNA (50 $\mathrm{ng} / \mu \mathrm{l})$. The reaction procedure was as follows: an initial denaturing at $95^{\circ} \mathrm{C}$ for $5 \mathrm{~min}$, followed by 5 cycles of three steps: $30 \mathrm{~s}$ denaturing at $95^{\circ} \mathrm{C}, 30 \mathrm{~s}$ annealing at $37^{\circ} \mathrm{C}$ and $1 \mathrm{~min}$ elongation at $72^{\circ} \mathrm{C}$. In the following 35 cycles, the annealing temperature was increased to $50^{\circ} \mathrm{C}$, with a final elongation step at $72^{\circ} \mathrm{C}$ for $8 \mathrm{~min}$. Amplification products were separated by electrophoresis in $12 \%$ polyacrylamide gel and silver stained (Brant, 1991). Each reaction was repeated at least two times to test for reproductively of the selected markers.

\section{Data scoring and statistical analysis}

DNA fragments were scored as "1" and "0", where "1" stands for the presence and " 0 " stands for the absence of each SRAP 


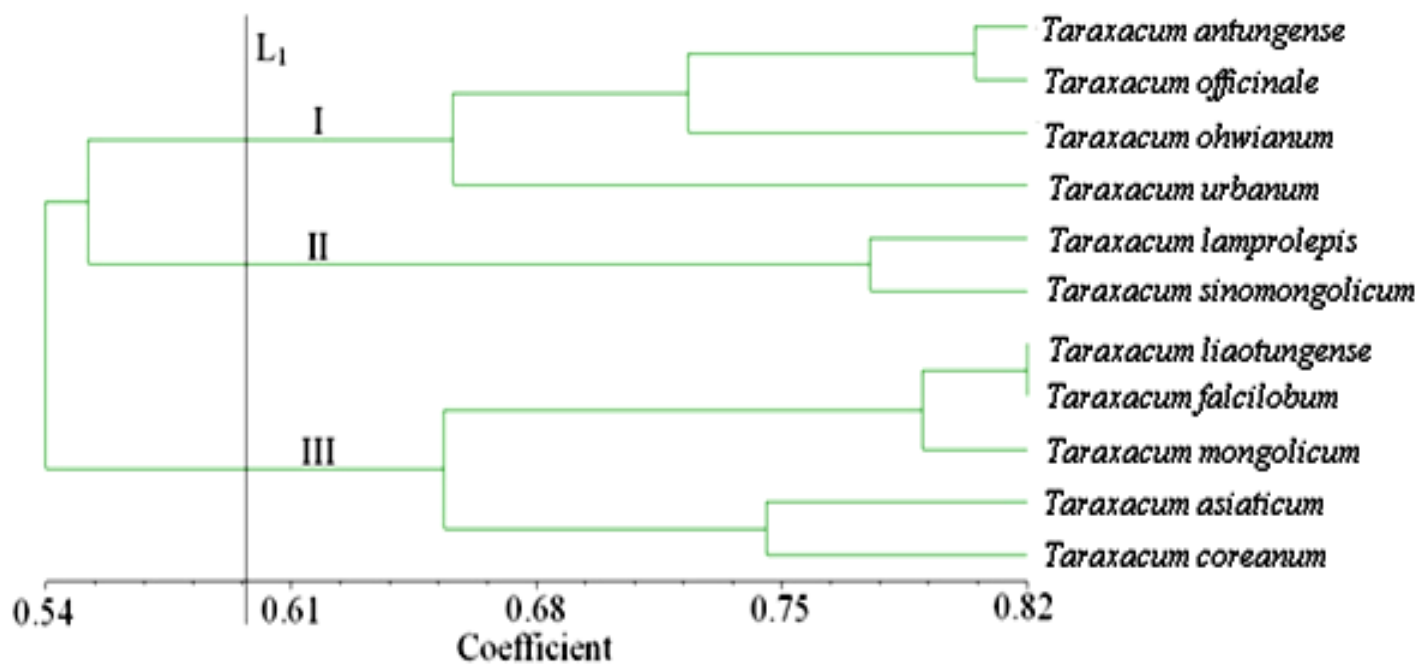

Figure 1. Dendrogram of 11 populations based on morphological data.

fragment. The fragment data were transformed into a binary (1/0) data matrix. Genetic similarity was analyzed using Nei and Li's index of similarity with the formula:

Similarity $=\mathrm{N}_{\mathrm{ab}} / \mathrm{N}_{\mathrm{a}}+\mathrm{N}_{\mathrm{b}}$

Where, $\mathrm{N}_{\mathrm{ab}}$ represents the number of fragments shared by fragment $a$ and $b ; N_{a}$ represents amplified fragments in sample $a ; N_{b}$ represents amplified fragments in sample $b$ (Nei and $\mathrm{Li}, 1979)$. The inbreds were grouped using the un-weighted pair group method (UPGMA) (Sokal and Michener, 1958). Cluster analysis was performed on the similarity matrix in order to generate phenograms using the NTSYS-pc 2.1 software (Rohlf, 2000).

\section{RESULTS}

\section{Morphological analysis}

Relationships between the 34 morphological parameters revealed by cluster analyses are presented in Figure 1. Three main clusters can be observed. The first cluster includes the Taraxacum antungense, Taraxacum officinale, Taraxacum ohwianum and Taraxacum urbanum. The second cluster comprises Taraxacum lamprolepis and Taraxacum sinomongolicum. The third one includes Taraxacum liaotungense, Taraxacum falcilobum, Taraxacum mongolicum, Taraxacum asiaticum and Taraxacum coreanum. T. liaotungense had the most closely genetic relationship with $T$. falcilobum (GS $=0.82)$. In addition, $T$. antungense and $T$. officinale also showed close relationship $(G S=0.80)$. The results illustrate that the first cluster had closer relationship with the third cluster.

\section{Polymorphism detected by SRAP}

A total of 110 primer combinations were assayed on three representative populations of taraxacum. Primers banding patterns that were difficult to score and those that failed to amplify consistently were excluded. Consequently, 20 primer combinations, which consistently produced well defined bands, were selected to show polymorphisms (Table 3). A total of 831 bands were observed, among which 795 were polymorphic (95.7\%), ranging between 22 and 55 per primer combination, with an average of 39.75 polymorphic bands per pair of primers. The combination of primers showing most polymorphism was the combination of EM3-ME4, EM4ME3 and EM4-ME4 (100\%), whereas the primer combination of EM5-ME3 had the lowest polymorphism (81\%).

The bands that were unique could be used as the special marker to distinguish the genotypes of different taraxacum. For instance, in combinations of primer P6, material 7 and material 9 had two special amplified bands, respectively. In addition material 3 and material 11 showed one special amplified band, respectively (Figure 2 , the special band was showed as red arrow); in the primer combination of P8, material 2 had one special band and material 6 showed two special bands (Figure 2). In the combinations of primer P10, material 9 and material 10 had two special bands, respectively (Figure 2). The special bands observed might be used to identify the genotypes of taraxacums.

\section{Genetic relationships}

A dendrogram based on the similarity coefficient of the 11 populations was constructed (Figure 3). Three major clusters can be identified at different similarity level. The first cluster (I) includes 5 populations of taraxacum: $T$. mongolicum, T. liaotungense, T. asiaticum, T. falcilobum and $T$. coreanum. T. mongolicum was closely related with 
Table 3. Polymorphism based on 20 primer combinations.

\begin{tabular}{llcccc}
\hline $\begin{array}{l}\text { Primer } \\
\text { code }\end{array}$ & $\begin{array}{l}\text { Primer } \\
\text { combination }\end{array}$ & $\begin{array}{c}\text { Number of } \\
\text { amplified band }\end{array}$ & $\begin{array}{c}\text { Number of } \\
\text { polymorphism band }\end{array}$ & $\begin{array}{c}\text { Percentage of } \\
\text { polymorphic band (\%) }\end{array}$ & $\begin{array}{c}\text { Polymorphism } \\
\text { information content }\end{array}$ \\
\hline P1 & EM1-ME1 & 42 & 41 & 0.97619 & 0.96412 \\
P2 & EM1-ME2 & 51 & 50 & 0.980392 & 0.971445 \\
P3 & EM1-ME3 & 36 & 33 & 0.916667 & 0.958293 \\
P4 & EM1-ME4 & 44 & 43 & 0.977273 & 0.969298 \\
P5 & EM2-ME1 & 35 & 32 & 0.914286 & 0.955748 \\
P6 & EM2-ME2 & 50 & 48 & 0.960000 & 0.969944 \\
P7 & EM2-ME3 & 45 & 42 & 0.933333 & 0.966598 \\
P8 & EM2-ME4 & 55 & 50 & 0.909091 & 0.975985 \\
P9 & EM3-ME1 & 22 & 21 & 0.954545 & 0.929031 \\
P10 & EM3-ME2 & 49 & 47 & 0.959184 & 0.969325 \\
P11 & EM3-ME3 & 46 & 45 & 0.978261 & 0.969941 \\
P12 & EM3-ME4 & 48 & 48 & 1.00000 & 0.968352 \\
P13 & EM4-ME1 & 47 & 46 & 0.978723 & 0.965883 \\
P14 & EM4-ME2 & 40 & 39 & 0.97500 & 0.965873 \\
P15 & EM4-ME3 & 38 & 38 & 1.000000 & 0.959897 \\
P16 & EM4-ME4 & 44 & 44 & 1.00000 & 0.964419 \\
P17 & EM5-ME1 & 35 & 34 & 0.971429 & 0.960081 \\
P18 & EM5-ME2 & 35 & 32 & 0.914286 & 0.96131 \\
P19 & EM5-ME3 & 32 & 26 & 0.812500 & 0.957403 \\
P20 & EM5-ME4 & 37 & 36 & 0.972973 & 0.964736 \\
& Total & 831 & 795 & & \\
& Mean & 41.55 & 39.75 & 0.956679 & 0.963384 \\
\hline
\end{tabular}

T. liaotungense at the similarity level of 0.74 . The second cluster (II) consists of $T$. lamprolepis and $T$. sinomongolicum at the similarity level of 0.73 , which were distinctly separated from other clusters. The third cluster (III) includes 4 taraxacum populations: $T$. antungense (Shenyang, Liaoning), T. officinale (Panshi, Jilin) $T$. urbanum (Benxi, Liaoning) and T. ohwianum (Dandong, Liaoning). The similarity coefficient between $T$. officinale and $T$. urbanum was 0.72 .

\section{DISCUSSION}

SRAP marker may have various applications for genetic studies and practical breeding programs in taraxacum. The dominant SRAP markers could provide more accurate information on population genetic diversity than traditional methods. The results provide evidence that SRAP is an efficient approach suitable for taxonomic analysis of different populations of taraxacum.

Previous studies have demonstrated that in the genetic diversity analysis, the information derived from SRAP marker was more concordant to the morphological variability and to the evolutionary history of the morphotypes than that of other molecular markers (Ferriol et al., 2003). The results of SRAP in our study show good amplification, stability and productivity with easily found polymorphism in the populations of taraxacum plants. Similar clusters of taraxacum were identified with both the morphological and SRAP markers, indicating that it is possible to evaluate the genetic diversity with SRAP molecular marker.

Both of the two methods divided the 11 populations of taraxacum into three clusters, nonetheless, the results of cluster analyses from the morphological and SRAP markers showed some differences, which probably due to the two detecting system based on different criteria. It is likely that some of the polymorphic site detected by SRAP marker and shown as the morphological traits could hardly be observed by naked eyes or might be neglected. It remains unclear whether the polymorphic sites detected by SRAP makers reflected the morphologic traits in our study. We suggested that some specific combinations of primer be explored to unify the classification from the two markers.

In this study, the DNA-based SRAP marker was successfully used; which distinguished the genetic diversity of taraxacum from the molecular level. The SRAP marker was suitable for the molecular investigation of phylogenic relationships between different populations of taraxacum. This study revealed the utility of SRAP markers in detecting genetics diversity and relations among populations of taraxacum, which could be useful to identify, select and breed varieties of taraxacum. 


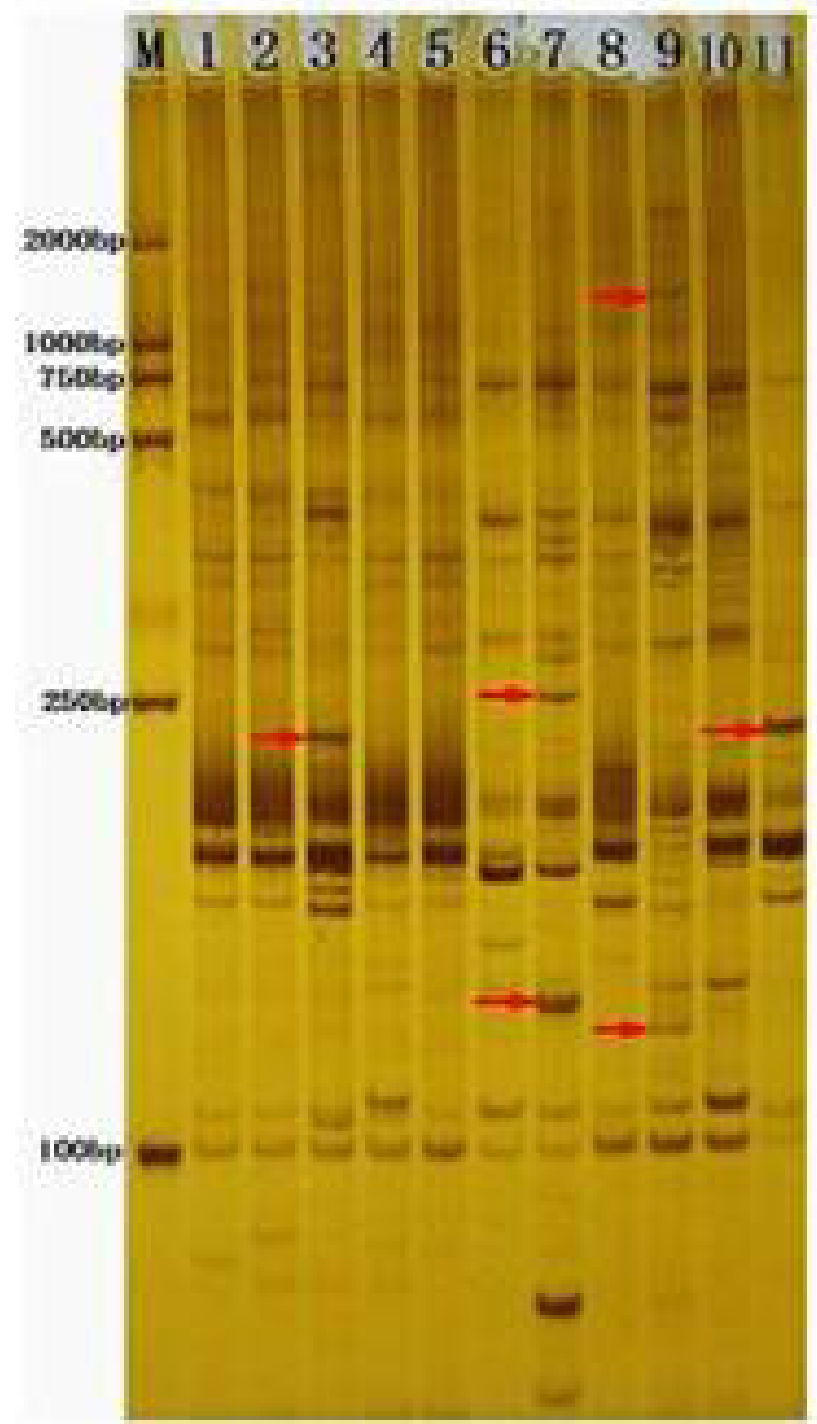

P6

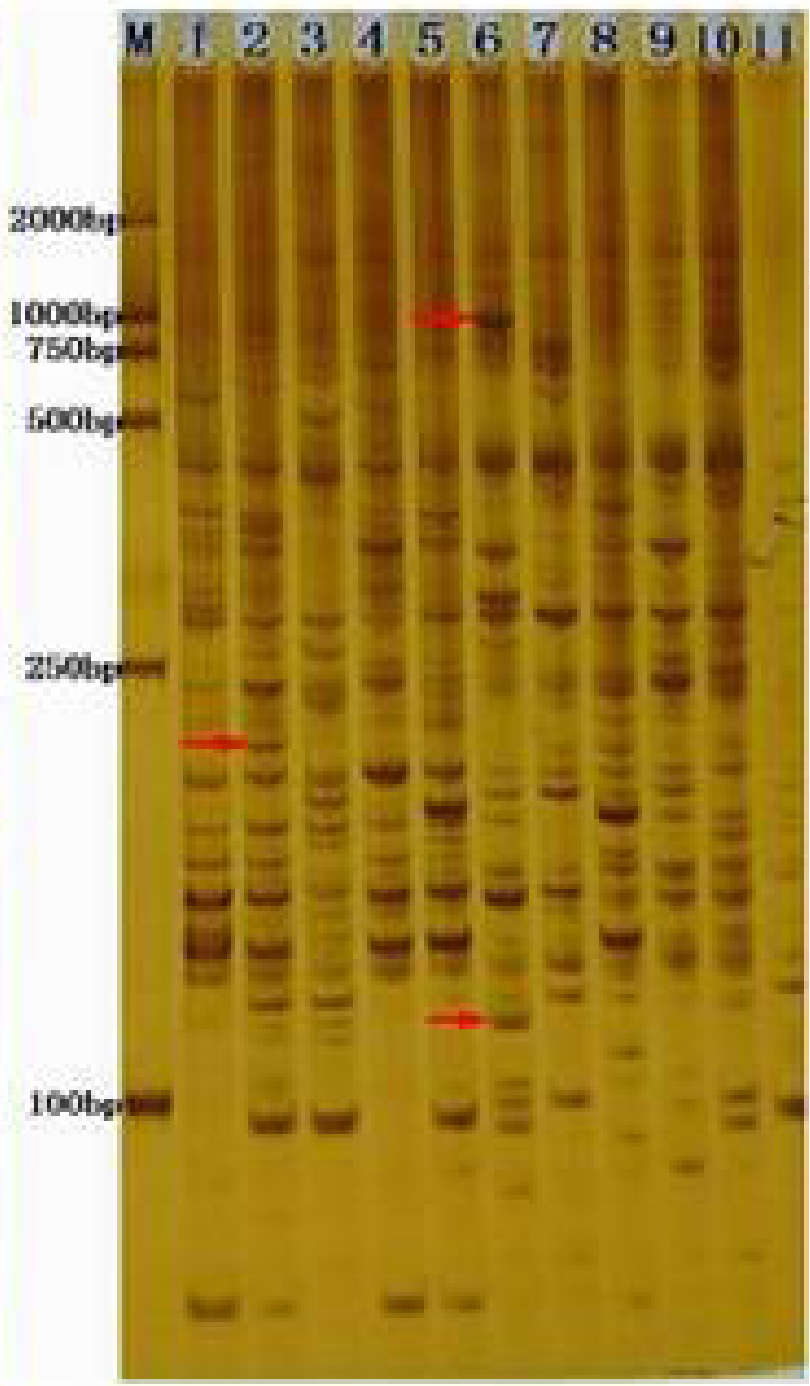

P8

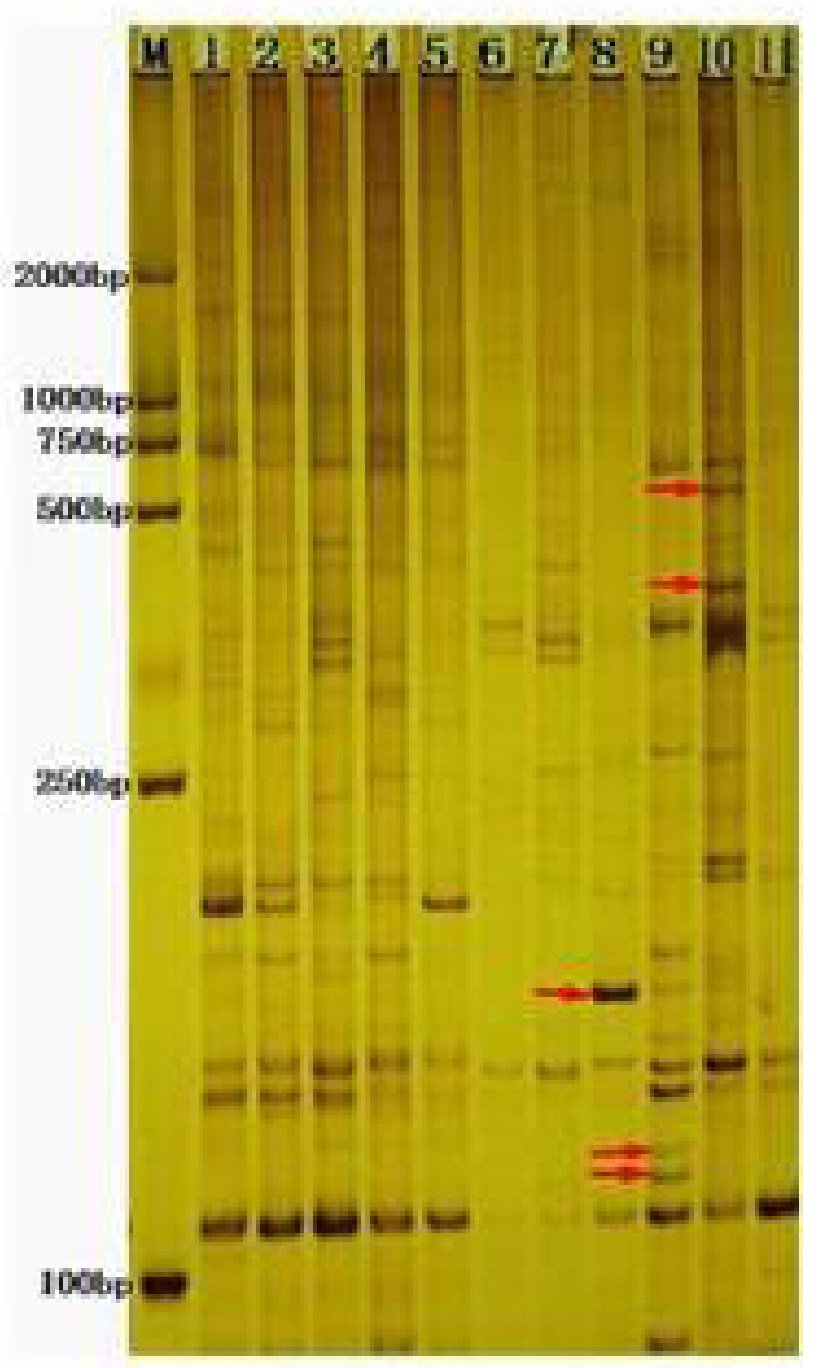

P10

Figure 2. Parts of the SRAP amplification profile of prime combination. The arrows are polymorphic bands, M is molecular markers; the number were the same as those in Table 1. 


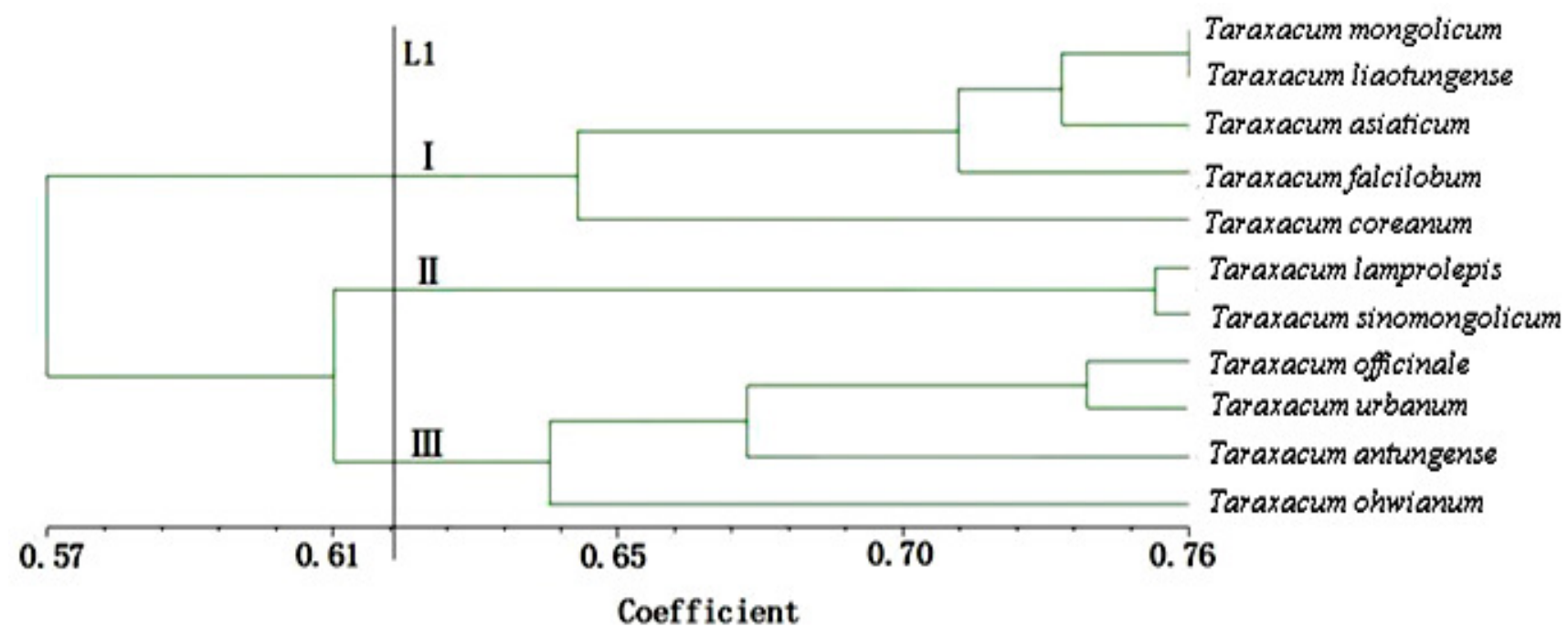

Figure 3. An un-weighted pair-group method with arithmetic averages (UPGMA) dendrogram of phylogenetic relationships among 11 populations based on similarity coefficients from the 20 SARP primer combinations. The resulting three clusters are labeled I, II and III.

\section{ACKNOWLEDGEMENT}

This study was supported by the Protection of wild plant resources-Project of the Ministry of Agriculture of China.

\section{REFERENCES}

Brant JB, Gustevo CA, Peter MG (1991). Fast and sensitive silver staining of DNA in polyacrylamide gels. Anal. Biochem. 196: 80-85.

Chen D, Peng R, Li L, Sun N, Zhong G, Cai Y (2009). Study on genetic diversity of Codonopsis tangshen by SRAP and ISSR markers. China J. Ch. Mater. Med. 3: 255-259.

Englbrecht CC, Freyhof J, Nolte A, Rassmann K, Schliewen U, Tautz D (2000). Phylogeography of thebullhead Cottus gobio (Pisces: Teleostei: Cottidae) suggests a pre-Pleistocene origin of the majorcentral European populations. Mol. Ecol. 9: 709-722.

Esposito MA, Martin EA, Cravero VP, Cointry E (2007). Characterization of pea accessions by SRAP's markers. Sci. Hort. 113: 329-335.

Ferriol M, Pic B, Nuez F (2003). Genetic diversity of a germplasm collection of Cucurbita pepo using SRAP and AFLP marker. Theor. Appl. Genet. 107: 271-282.

Ge XJ, Ling YR, Zhai DT (1998). A synopsis of Taraxacum F. H. wigg (compositae) from China. Bull. Botan. Res. 4: 377-397.

He FF, Yang ZP, Zhang ZS, Wang GX, Wang JC (2007). Genetic Diversity Analysis of Potato Germplasm by SRAP Makers. J. Agr. Biotechnol. 6: 1001-1005.

Jiang SK, Ma H, Liu J, He J, Guo ZF, Chen LJ, Zhong M, Zhang LJ, Wang XY, Zhang L (2007). Genetic Diversity in Maize Inbred lines Revealed by SRAP Marker. Mol. PI. Breed. 3: 412- 416.

Li G, Quiros CF (2001). Sequence-related amplified polymorphism (SRAP), a new marker system based on a simple PCR reaction: its application to mapping and gene tagging in Brassica. Theor. Appl. Genet. 103: 455-461.

Li JY(2004). Northeast herb Zhi. Book IX. 95-231.

Lin ZX, Zhang XL, Nie YC, He DH, Wu MQ (2003). Ch. Sc. Bull. 48: 2064-2068.

Liu JM, Wang L, Geng YP, Wang QB, Luo LJ, Zhong Y (2006). Genetic diversity and population structure of Lamiophlomis rotate (Lamiaceae), an endemic species of Qinghai-Tibet Plateau. Genetica. 128: 385-394.
Mutlu N, Boyaci FH, Abak MG (2008). Development of SRAP, SRAPRGA, RAPD and SCAR markers linked with a Fusarium wilt resistance gene in eggplant. Theor. Appl. Genet. 8: 1303-1312.

Nei M, Li WH (1979). Mathematical model for studying genetic variation in terms of restriction endonucleases. Proc. Natl. Acad. Sci. USA. 76: 5269-5273.

Qian D, Huang LQ, Cui GH, Chen M (2009). Study on genetic relationship of Astragalus membranaceus var mongholicus in different producing area using SRAP. China J. Ch. Mater. Med. 4: 382-385.

Ren Y, Wang DY, Zhang YD, Li Y, Wang HM (2004). Optimization of SRAP-PCR in Hot Pepper (Capsicum annuum L). Mol. PI. Breed. 5 : 689-693.

Riaz A, Li G, Quresh Z, Swati MS, Quiros CF (2001). Genetic diversity of oil seed Brassica napus inbred lines based on sequencerelated amplified polymorphism and its relation to hybrid performance. Plant Breed. 120 (5): 411-415.

Rohlf FJ (2000). NTSYS-pc: Numerical Taxonomy and Multivariate Analysis System. Version 2.1. Exeter Publications, New York, USA, p189.

Sokal RR, Michener CD (1958). A statistical method for evaluating systematic relationships. Univ. Kansas Sci. Bull. 28: 1409-1438.

Whitehead A, Anderson SL, Kuivila KM, Roach JL, May B(2003). Genetic variation among interconnected populations of Catostomus occidentalis: implications for distinguishing impacts of contaminants from biogeographical structuring. Mol. Ecol. 12: 2817-2833.

Wu GY, Pan HZ (1999). Experimental databook of biochemistry and molecular biology. Beijing Sc. Sinica. 25-36.

Zhong J, Wang KQ, Ping Z, Wei J, Xiong XY (2010). An Analysis of Genetic Diversity of Houttuynia cordata Thunb. Population by SRAP Mol. Markers. Plant Physiol.Commun. 3: 210-221 